\title{
Evolutionary Dynamics of Mandatory Lane Changing for Bus Exiting
}

\author{
Ronghan Yao $\left(\mathbb{D}\right.$, Xiaojing Du $\left(\mathbb{D}\right.$, Wenyan Qi $\left(\mathbb{D}\right.$, and Li Sun $\mathbb{D}^{\circ}$ \\ School of Transportation and Logistics, Dalian University of Technology, Dalian 116024, China \\ Correspondence should be addressed to Xiaojing Du; duxiaojing@mail.dlut.edu.cn
}

Received 8 September 2021; Revised 8 November 2021; Accepted 15 November 2021; Published 30 December 2021

Academic Editor: Daxin Tian

Copyright (c) 2021 Ronghan Yao et al. This is an open access article distributed under the Creative Commons Attribution License, which permits unrestricted use, distribution, and reproduction in any medium, provided the original work is properly cited.

\begin{abstract}
With the development of the connected autonomous bus, the interactions between the bus and social vehicle during the mandatory lane changing for bus exiting become more diverse and complex. This research investigates the evolutionary dynamics of behavioral decision-making for the bus and social vehicle in different scenarios. The evolutionary game model for the connected autonomous bus and social vehicle is built, as do the human-driven bus and social vehicle, and the connected autonomous bus under different penetration rates and social vehicle. The results of numerical experiments reveal that the connected autonomous bus chooses to change lanes in most instances, and the strategies of the human-driven bus show conservative tendencies. Such tendencies are weakened when the connected autonomous bus and human-driven bus are mixed. As for the social vehicle in different scenarios, the strategies that balance overall traffic safety and efficiency are promoted. This research provides some references for intelligent decision-making of lane changing in urban public transportation.
\end{abstract}

\section{Introduction}

Mandatory lane changing for bus exiting is one essential part in the daily operations of the human-driven bus. In this lanechanging process, the bus driver must make reasonable behavioral decision-making to guarantee driving safety and efficiency $[1,2]$. With the development of the connected autonomous bus ( $\mathrm{CAB}$ hereon), there is no longer only the human-driven bus (HDB hereon) on urban roads. The decision-making of the bus may be changed due to various characteristics of different kinds of buses. Meanwhile, it is inevitable that $\mathrm{HDB}$ and $\mathrm{CAB}$ are mixed with different penetration rates for a long time in the total transition from $\mathrm{HDB}$ to $\mathrm{CAB}$, which makes the behavioral decision-making more complicated. Therefore, it has great significance to explore the behavioral decision-making in the mandatory lane changing for bus exiting in different scenarios, i.e., $C A B$, $\mathrm{HDB}$, and $\mathrm{CAB}$ under different penetration rates (BPR hereon).

During the decision-making process, whether the bus is human-driven or connected and autonomous, the behavioral decision-making of the bus will affect and be affected by that of the social vehicle on the adjacent lane. In order to investigate the interactions between different vehicles, the game theory is utilized for lane-changing issues. For humandriven vehicles, Kita first built a mixed strategy game model for vehicles on the highway according to the time to collision [3]. On this basis, the risk conflicts [4], safety and efficiency of lane changing [5-7], driving space [8], and lane-changing requirements [9] were considered in the mixed strategy lanechanging game models on the highway or urban streets. Kang and Rakha [10] proposed a repeated game model by calculating the accumulated payoffs for the players so as to explore the impacts of the history of the players' decisionmaking on the choice of strategies. The conclusions reveal that the consideration of previous decision-making can improve the accuracy of behavioral predictions. For the lanechanging game in a connected environment, the Harsanyi theory [11] was employed to describe the uncertainty of the intention of lane changing [12] and driving environment [13]. In a mixed traffic flow composed of human-driven and autonomous vehicles, some scholars adopted the mixed strategy game [14], Stackelberg game [15], and cooperative game [16] to describe the lane-changing issue.

In all the lane-changing game models discussed above, both the connected autonomous vehicle and the human- 
driven vehicle driver are assumed to be perfect rational players. That is to say, the vehicle and the driver can completely eliminate all external and internal interference, e.g., insufficient computing skills and driver heterogeneity, and make the best decision-making for themselves. However, the assumption of perfect rationality occurs with a very small probability or even never occurs in the lane-changing process. In practice, although the connected autonomous vehicles can obtain driving information in time, they have limited information perception capabilities for the roads and surrounding vehicles $[17,18]$, which lead to imperfect rationality in the decision-making. As for human-driven vehicle drivers, they cannot respond to others' decisionmaking and the changes in the surrounding environment with perfect rationality because of physiological and psychological factors $[19,20]$. Therefore, the assumption of perfect rationality in the basic-form game theory cannot truly and comprehensively describe the decision-making process.

In view of the hypothesis flaw of perfect rationality in the basic-form game theory, the evolutionary game theory was proposed. In the evolutionary game theory, the research objects are populations which are considered to have bounded rationality and cannot obtain equilibrium through only one static game. The game, therefore, is a repeated and dynamic process, in which strategies that represent greater payoffs will be spread among game populations before game equilibria are formed [21]. The evolutionary game theory has been utilized to solve the problems in the field of transportation, such as travel mode choice [22-24], conflicts between pedestrian and vehicle [25], and the safety regulation of railway transportation $[26,27]$. However, the literature that focuses on the evolutionary game theory-based lane-changing models is limited [28]. Lwamura and Tanimoto discussed the interactions between social vehicles on the highway by applying the evolutionary game theory, and the result implied that a social dilemma was hidden in the lane changing [29]. On this basis, Tanimoto et al. analyzed the lane-changing behaviors of social vehicles in different scenarios, such as human-driven and autonomous driving environments [30]. Compared with the lane changing of social vehicles, different characteristics exist in the mandatory lane changing for bus exiting, e.g., larger traffic delay caused by mass passengers and discomfort caused by excessive acceleration. These unique characteristics of the mandatory lane changing for bus exiting should be further considered to make the models more accurate.

The objective of this paper is to formulate new models of mandatory lane changing for bus exiting in different scenarios. More specifically, the evolutionary game theory is applied to demonstrate the interactions between different kinds of buses (CAB, HDB, and BPR) and social vehicles. The bus and the social vehicle are regarded as populations which constantly exchange information and experience for learning and optimizing decision-making. Accordingly, the evolutionary mechanism of the strategies adopted by the populations is investigated based on the stable-state analysis and the numerical experiments.
The remainder of this paper is organized as follows. The models formulated in this paper, including the assumptions, and replicator dynamics are firstly introduced in Section 2. Then, the equilibrium points are calculated and the stable states of the dynamic game systems in different conditions are analyzed in Section 3. The design and results of numerical experiments are elaborated in Section 4 Finally, the main conclusions of this paper and the recommendations for future research are summarized in Section 5.

\section{Models}

In order to deal with different scenarios when buses exit from bus bays, three evolutionary game models, i.e., the model for $\mathrm{CAB}$ and the social vehicle (Model 1), the model for HDB and the social vehicle (Model 2), and the model for BPR and the social vehicle (Model 3) will be developed, respectively. The scenarios of the lane-changing game in Models 1 and 2 are illustrated in Figure 1. The scenario of the lane-changing game in Model 3 is a mix of Models 1 and 2 via different penetration rates.

2.1. Assumptions. The assumptions of the mandatory lane changing for bus exiting are as follows.

2.1.1. Populations. There are always two populations in Models 1, 2, and 3. The populations are the bus that has finished service and is about to exit from the bus bay stop and the social vehicle on the adjacent lane. The scenarios in which buses are connected and autonomous in Model 1, human-driven in Model 2, and mixed under different penetration rates in Model 3 are considered in this paper, respectively. The level of the connected autonomous bus is assumed to be Level 5. However, in the long-term development of autonomous social vehicles, it is very likely that high-level autonomous social vehicles have low-penetration rates because of the expensive costs for the drivers [31]. According to the taxonomy of driving automation for vehicles formulated by the Chinese government, humandriven vehicles still play a leading role in the low level of autonomous driving [32]. Therefore, the social vehicles researched in this paper are all assumed to be human-driven vehicles in all the three models.

2.1.2. Strategies. The buses, including $\mathrm{CAB}, \mathrm{HDB}$, and $\mathrm{BPR}$, have two strategies, i.e., change lanes and do not change lanes. The human-driven social vehicle (HDS hereon) also has two strategies, i.e., give way and do not give way. Therefore, there are totally four strategy profiles between the bus and human-driven social vehicle, i.e., \{(change lanes, give way), (change lanes, do not give way), (do not change lanes, give way), (do not change lanes, do not give way)\}.

2.1.3. Payoffs. The two populations in Models 1,2 , and 3 may obtain the time and safety benefit and suffer the time and safety loss. For example, when the bus waits at the bus bay stop, its safety is completely guaranteed, but it has to 


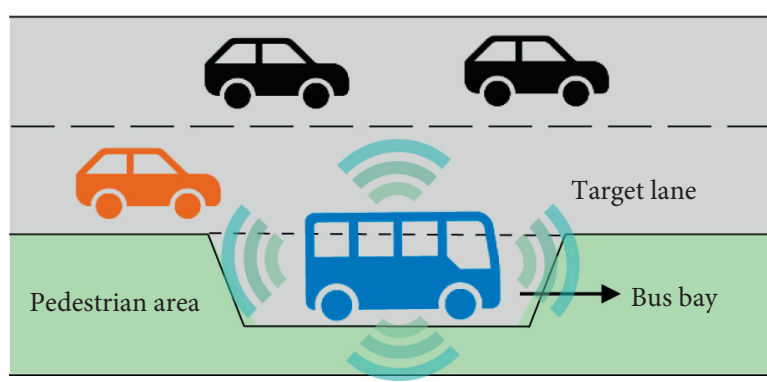

(a)

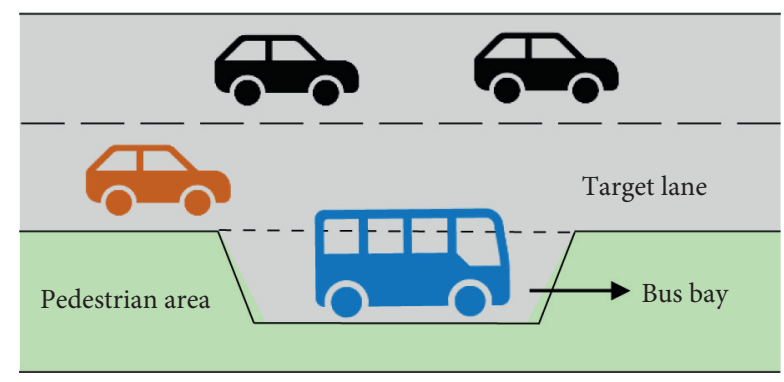

(b)

Figure 1: Scenarios of lane changing game in (a) Model 1 and (b) Model 2.

spend time waiting for HDS to pass through. In such a process, the bus gets safety, but loses time. Moreover, compared with $\mathrm{CAB}, \mathrm{HDB}$ is not equipped with the driving assistance system and precise control system, so it is likely to cause noncollision injuries to the passengers because of excessive start acceleration [33]. Therefore, the extra comfort loss of changing lanes for HDB needs to be considered in Models 2 and 3. The variables and their meanings are listed in Table 1. The subscript $C$ of the variable means the strategy of changing lanes for the bus, and the subscript NC means the strategy of not changing lanes for the bus. As for HDS, the subscript $\mathrm{G}$ represents the strategy of giving way, and the subscript NG represents the strategy of not giving way.

2.1.4. Proportions of strategies. The proportion of the lanechanging strategy for $\mathrm{CAB}$ is $x_{1}$, that for $\mathrm{HDB}$ is $x_{2}$, and that for BPR is $x_{3},\left(x_{1}, x_{2}, x_{3} \in[0,1]\right)$. Accordingly, the proportions of not changing lane strategy for $\mathrm{CAB}, \mathrm{HDB}$, and BPR are $1-x_{1}, 1-x_{2}$, and $1-x_{3}$, respectively. The proportions of giving way strategy for HDS in Models 1, 2, and 3 are all $y(y \in[0,1])$, and those of not giving way strategy are all $1-y$, since the social vehicles in different scenarios are the same, i.e., HDS.

2.2. Replicator Dynamics. Based on the above assumptions, the payoff matrix in Model 1 is shown in Table 2. The strategy profiles of changing lanes and giving way, and not changing lanes and not giving way balance traffic safety and efficiency, that is, one population's safety and the other's efficiency, can be ensured. In the strategy profile of not changing lanes and giving way, although the safety of the bus and HDS can be totally guaranteed, more traffic delays will be generated. When both the bus and HDS choose aggressive strategies, i.e., change lanes and do not give way, negative effects will be easily caused by collisions.

According to the payoff matrix shown in Table 2 and the proportions of giving way and not giving way strategies for HDS, the expected payoffs of the strategies of changing lanes and not changing lanes for $C A B$, which are denoted by $E_{\mathrm{CAB}-B_{1}}$ and $E_{\mathrm{CAB}-B_{2}}$, respectively, can be expressed as
TABLE 1: Variables and their meanings.

\begin{tabular}{|c|c|c|c|}
\hline Population & Variable & Meaning of the variable & Note \\
\hline \multirow{4}{*}{$\begin{array}{l}\mathrm{CAB} \text { and } \\
\mathrm{HDB}\end{array}$} & $M_{\mathrm{C}}$ & Safety loss of changing lanes & $M_{\mathrm{C}}>0$ \\
\hline & $R_{\mathrm{C}}$ & $\begin{array}{c}\text { Time benefit of changing } \\
\text { lanes }\end{array}$ & $R_{\mathrm{C}}>0$ \\
\hline & $J_{\mathrm{NC}}$ & $\begin{array}{c}\text { Safety benefit of not changing } \\
\text { lanes }\end{array}$ & $J_{\mathrm{NC}}>0$ \\
\hline & $K_{\mathrm{NC}}$ & $\begin{array}{c}\text { Time loss of not changing } \\
\text { lanes }\end{array}$ & $K_{\mathrm{NC}}>0$ \\
\hline $\mathrm{HDB}$ & $T_{\mathrm{C}}$ & $\begin{array}{l}\text { Extra comfort loss of } \\
\text { changing lanes }\end{array}$ & $T_{\mathrm{C}}>0$ \\
\hline \multirow{3}{*}{ HDS } & $\begin{array}{l}J_{\mathrm{G}} \\
K_{\mathrm{G}}\end{array}$ & $\begin{array}{l}\text { Safety benefit of giving way } \\
\text { Time loss of giving way }\end{array}$ & $\begin{array}{l}J_{\mathrm{G}}>0 \\
K_{G}>0\end{array}$ \\
\hline & $M_{\mathrm{NG}}^{\mathrm{G}}$ & Safety loss of not giving way & $M_{\mathrm{NG}}^{\mathrm{G}}>0$ \\
\hline & $R_{\mathrm{NG}}$ & $\begin{array}{c}\text { Time benefit of not giving } \\
\text { way }\end{array}$ & $R_{\mathrm{NG}}>0$ \\
\hline
\end{tabular}

TABle 2: Payoff matrix for CAB and HDS.

\begin{tabular}{lcc}
\hline \multirow{2}{*}{$\mathrm{CAB}$} & \multicolumn{2}{c}{ HDS } \\
& Give way & Do not give way \\
\hline Change lanes & $R_{\mathrm{C}}, J_{\mathrm{G}}$ & $-M_{\mathrm{C}},-M_{\mathrm{NG}}$ \\
Do not change lanes & $J_{\mathrm{NC}}-K_{\mathrm{NC}}, J_{\mathrm{G}}-K_{\mathrm{G}}$ & $J_{\mathrm{NC}}, R_{\mathrm{NG}}$ \\
\hline
\end{tabular}

$$
\begin{aligned}
E_{\mathrm{CAB}-B_{1}} & =y \cdot R_{\mathrm{C}}+(1-y) \cdot\left(-M_{\mathrm{C}}\right) \\
& =y \cdot\left(M_{\mathrm{C}}+R_{\mathrm{C}}\right)-M_{\mathrm{C}}, \\
E_{\mathrm{CAB}-B_{2}} & =y \cdot\left(J_{\mathrm{NC}}-K_{\mathrm{NC}}\right)+(1-y) \cdot J_{\mathrm{NC}} \\
& =-y \cdot K_{\mathrm{NC}}+J_{\mathrm{NC}} .
\end{aligned}
$$

Then, the average expected payoff for $\mathrm{CAB}$, which is denoted by $\bar{E}_{\mathrm{CAB}}$, can be determined by

$$
\begin{aligned}
\bar{E}_{\mathrm{CAB}}= & x_{1} \cdot E_{\mathrm{CAB}-B_{1}}+\left(1-x_{1}\right) \cdot E_{\mathrm{CAB}-B_{2}} \\
= & x_{1} y \cdot\left(M_{\mathrm{C}}+R_{\mathrm{C}}+K_{\mathrm{NC}}\right)-x_{1} \cdot\left(M_{\mathrm{C}}+J_{\mathrm{NC}}\right) \\
& -y \cdot K_{\mathrm{NC}}+J_{\mathrm{NC}} .
\end{aligned}
$$

Based on the expected payoffs of the strategies for $\mathrm{CAB}$, the replicator dynamics equation for $\mathrm{CAB}$ can be written as 


$$
F_{\mathrm{CAB}}\left(x_{1}, y\right)=x_{1}\left(1-x_{1}\right)\left[y \cdot\left(M_{\mathrm{C}}+R_{\mathrm{C}}+K_{\mathrm{NC}}\right)-M_{\mathrm{C}}-J_{\mathrm{NC}}\right] .
$$

Similarly, the expected payoffs of the strategies of giving way and not giving way for HDS, which are denoted by $E_{\mathrm{HDS}-S_{1}}$ and $E_{\mathrm{HDS}-S_{2}}$, and the average expected payoff for HDS, which is denoted by $\bar{E}_{\mathrm{HDS}}$, can be given by

$$
\begin{aligned}
E_{\mathrm{HDS}-S_{1}}= & x_{1} \cdot J_{\mathrm{G}}+\left(1-x_{1}\right) \cdot\left(J_{\mathrm{G}}-K_{\mathrm{G}}\right) \\
= & x_{1} \cdot K_{\mathrm{G}}+J_{\mathrm{G}}-K_{\mathrm{G}}, \\
E_{\mathrm{HDS}-S_{2}}= & x_{1} \cdot\left(-M_{\mathrm{NG}}\right)+\left(1-x_{1}\right) \cdot R_{\mathrm{NG}} \\
= & -x_{1} \cdot\left(M_{\mathrm{NG}}+R_{\mathrm{NG}}\right)+R_{\mathrm{NG}}, \\
\bar{E}_{\mathrm{HDS}}= & y \cdot E_{\mathrm{HDS}-S_{1}}+(1-y) \cdot E_{\mathrm{HDS}-S_{2}} \\
= & x_{1} y \cdot\left(K_{\mathrm{G}}+M_{\mathrm{NG}}+R_{\mathrm{NG}}\right)-x_{1} \cdot\left(M_{\mathrm{NG}}+R_{\mathrm{NG}}\right) \\
& +y \cdot\left(J_{\mathrm{G}}-K_{\mathrm{G}}-R_{\mathrm{NG}}\right)+R_{\mathrm{NG}} .
\end{aligned}
$$

Thus, based on the expected payoffs of the strategies for HDS, the replicator dynamics equation for HDS is

$$
\begin{aligned}
F_{\mathrm{HDS}}\left(x_{1}, y\right)= & y(1-y)\left[x_{1} \cdot\left(K_{\mathrm{G}}+M_{\mathrm{NG}}+R_{\mathrm{NG}}\right)\right. \\
& \left.+J_{\mathrm{G}}-K_{\mathrm{G}}-R_{\mathrm{NG}}\right] .
\end{aligned}
$$

The expected payoffs of the strategies for HDS will not be discussed again in the following, since the payoffs and replicator dynamics for HDS involved in different scenarios are the same, i.e., $F_{\mathrm{HDS}}\left(x_{1}, y\right)=F_{\mathrm{HDS}}\left(x_{2}, y\right)=F_{\mathrm{HDS}}\left(x_{3}, y\right)$. The expected payoffs for HDB and those for BPR will be analyzed in detail in Models 2 and 3.

Unlike $\mathrm{CAB}$, which can provide sufficient comfort experience for passengers, the smooth start of HDB is largely dependent on the drivers' experience, which may cause passengers' noncollision injuries in emergency situations. Therefore, on the basis of the payoff matrix for $\mathrm{CAB}$ and HDS, extra comfort loss is considered in the game between HDB and HDS when the strategy of changing lanes is chosen for bus exiting. The payoff matrix for HDB and HDS is listed in Table 3.

In accordance with the payoff matrix listed in Table 3 and the proportions of giving way and not giving way strategies for HDS, the expected payoffs of the strategies of changing lanes and not changing lanes, and the average expected payoff for $\mathrm{HDB}$, which are denoted by $E_{\mathrm{HDB}-B_{1}}, E_{\mathrm{HDB}-B_{2}}$, and $\bar{E}_{\mathrm{HDB}}$, can be calculated as

$$
\begin{aligned}
E_{\mathrm{HDB}-B_{1}}= & y \cdot\left(R_{\mathrm{C}}-T_{\mathrm{C}}\right)+(1-y) \cdot\left(-M_{\mathrm{C}}-T_{\mathrm{C}}\right) \\
= & y \cdot\left(M_{\mathrm{C}}+R_{\mathrm{C}}\right)-M_{\mathrm{C}}-T_{\mathrm{C}}, \\
E_{\mathrm{HDB}-B_{2}}= & y \cdot\left(J_{\mathrm{NC}}-K_{\mathrm{NC}}\right)+(1-y) \cdot J_{\mathrm{NC}} \\
= & -y \cdot K_{\mathrm{NC}}+J_{\mathrm{NC}}, \\
\bar{E}_{\mathrm{HDB}}= & x_{2} \cdot E_{\mathrm{HDB}-B_{1}}+\left(1-x_{2}\right) \cdot E_{\mathrm{HDB}-B_{2}} \\
= & x_{2} y \cdot\left(M_{\mathrm{C}}+R_{\mathrm{C}}+K_{\mathrm{NC}}\right) \\
& -x_{2} \cdot\left(M_{\mathrm{C}}+T_{\mathrm{C}}+J_{\mathrm{NC}}\right)-y \cdot K_{\mathrm{NC}}+J_{\mathrm{NC}} .
\end{aligned}
$$

Based on the expected payoffs of the strategies for HDB, the replicator dynamics equation for HDB is

$$
\begin{aligned}
F_{\mathrm{HDB}}\left(x_{2}, y\right)= & x_{2}\left(1-x_{2}\right)\left[y \cdot\left(M_{\mathrm{C}}+R_{\mathrm{C}}+K_{\mathrm{NC}}\right)\right. \\
& \left.-M_{\mathrm{C}}-T_{\mathrm{C}}-J_{\mathrm{NC}}\right] .
\end{aligned}
$$

When BPR is considered, a kind of random combination of Models 1 and 2 will be generated. The probability of the occurrence of Model 1 or 2 depends on the penetration rate of the connected autonomous bus. Therefore, the payoff of each strategy profile in Model 3 can be seen as a random mix of the payoffs of the corresponding strategy profiles in Models 1 and 2 according to the penetration rate of the connected autonomous bus. The expected payoffs of the strategies of changing lanes and not changing lanes for BPR, which are denoted by $E_{\mathrm{BPR}-B_{1}}$ and $E_{\mathrm{BPR}-B_{2}}$, can be derived by

$$
\begin{aligned}
E_{\mathrm{BPR}-B_{1}} & =\alpha \cdot E_{\mathrm{CAB}-B_{1}}+(1-\alpha) \cdot E_{\mathrm{HDB}-B_{1}} \\
& =y \cdot\left(R_{\mathrm{C}}+M_{\mathrm{C}}\right)-M_{\mathrm{C}}-(1-\alpha) \cdot T_{\mathrm{C}}, \\
E_{\mathrm{BPR}-B_{2}} & =\alpha \cdot E_{\mathrm{CAB}-B_{2}}+(1-\alpha) \cdot E_{\mathrm{HDB}-B_{2}} \\
& =-y \cdot K_{\mathrm{NC}}+J_{\mathrm{NC}} .
\end{aligned}
$$

where $\alpha$ is the penetration rate of the connected autonomous bus.

Then, the average expected payoff for BPR, which is denoted by $\bar{E}_{\mathrm{BPR}}$, can be obtained as

$$
\begin{aligned}
\bar{E}_{\mathrm{BPR}}= & x_{3} \cdot E_{\mathrm{BPR}-B_{1}}+\left(1-x_{3}\right) \cdot E_{\mathrm{BPR}-B_{2}} \\
= & x_{3} y \cdot\left(R_{\mathrm{C}}+M_{\mathrm{C}}+K_{\mathrm{NC}}\right)-x_{3} \cdot\left[M_{\mathrm{C}}+(1-\alpha)\right. \\
& \left.\cdot T_{\mathrm{C}}+J_{\mathrm{NC}}\right]-y \cdot K_{\mathrm{NC}}+J_{\mathrm{NC}} .
\end{aligned}
$$

Based on the expected payoffs of the strategies for BPR, the replicator dynamics equation for BPR is

$$
\begin{aligned}
F_{\mathrm{BPR}}\left(x_{3}, y\right)= & x_{3}\left(1-x_{3}\right)\left[y \cdot\left(R_{\mathrm{C}}+M_{\mathrm{C}}+K_{\mathrm{NC}}\right)-M_{\mathrm{C}}\right. \\
& \left.-(1-\alpha) \cdot T_{\mathrm{C}}-J_{\mathrm{NC}}\right] .
\end{aligned}
$$

\section{Stable-State Analysis}

3.1. Calculation of Equilibrium Points. The replicator dynamics equations shown in equations (3) and (5), equations (7) and (5), and equations (10) and (5) form three differential equation sets about the proportions of lane-changing and giving way strategies. The equilibrium points of the dynamic game systems can be obtained through solving the following differential equation sets, namely,

$$
\begin{aligned}
& \left\{\begin{array}{l}
F_{\mathrm{CAB}}\left(x_{1}, y\right)=0, \\
F_{\mathrm{HDS}}\left(x_{1}, y\right)=0,
\end{array}\right. \\
& \left\{\begin{array}{l}
F_{\mathrm{HDB}}\left(x_{2}, y\right)=0, \\
F_{\mathrm{HDS}}\left(x_{2}, y\right)=0,
\end{array}\right. \\
& \left\{\begin{array}{l}
F_{\mathrm{BPR}}\left(x_{3}, y\right)=0, \\
F_{\mathrm{HDS}}\left(x_{3}, y\right)=0,
\end{array}\right. \\
& x_{1}, x_{2}, x_{3}, y \in[0,1] .
\end{aligned}
$$

Regardless of the chosen values of the variables, each differential equation set always has 4 sets of solutions, i.e., 
TABLE 3: Payoff matrix for HDB and HDS.

\begin{tabular}{lcr}
\hline HDB & HDS & Do not give way \\
\hline Change lanes & $R_{\mathrm{C}}-T_{\mathrm{C}}, J_{\mathrm{G}}$ & $-M_{\mathrm{C}}-T_{\mathrm{C}},-M_{\mathrm{NG}}$ \\
Do not change lanes & $J_{\mathrm{NC}}-K_{\mathrm{NC}}, J_{\mathrm{G}}-K_{\mathrm{G}}$ & $J_{\mathrm{NC}}, R_{\mathrm{NG}}$ \\
\hline
\end{tabular}

$(0,0),(0,1),(1,0)$, and $(1,1)$. In addition, when the proportions are guaranteed between 0 and 1 , there is another solution. For the game between CAB and HDS, when $J_{\mathrm{NC}}-K_{\mathrm{NC}}<R_{\mathrm{C}}$ and $J_{\mathrm{G}}-K_{\mathrm{G}}<R_{\mathrm{NG}}$, there is another solution for the differential equation set, that is, $\left(\left(K_{\mathrm{G}}-J_{\mathrm{G}}+R_{\mathrm{NG}}\right) /\left(K_{\mathrm{G}}\right.\right.$ $\left.\left.+M_{\mathrm{NG}}+R_{\mathrm{NG}}\right), \quad\left(M_{\mathrm{C}}+J_{\mathrm{NC}}\right) /\left(M_{\mathrm{C}}+R_{\mathrm{C}}+K_{\mathrm{NC}}\right)\right)$. For the game between $\mathrm{HDB}$ and HDS, when $J_{\mathrm{NC}}-K_{\mathrm{NC}}<R_{\mathrm{C}}-T_{\mathrm{C}}$ and $J_{\mathrm{G}}-K_{\mathrm{G}}<R_{\mathrm{NG}}$, another solution for the differential equation set is $\left(\left(K_{\mathrm{G}}-J_{\mathrm{G}}+R_{\mathrm{NG}}\right) /\left(K_{\mathrm{G}}+M_{\mathrm{NG}}+R_{\mathrm{NG}}\right)\right.$, $\left.\left(M_{\mathrm{C}}+T_{\mathrm{C}}+J_{\mathrm{NC}}\right) /\left(M_{\mathrm{C}}+R_{\mathrm{C}}+K_{\mathrm{NC}}\right)\right)$. In Model 3 where BPR is considered, when $J_{\mathrm{NC}}-K_{\mathrm{NC}}<R_{\mathrm{C}}-(1-\alpha) \cdot T_{\mathrm{C}}$ and $J_{\mathrm{G}}-K_{\mathrm{G}}<R_{\mathrm{NG}}$, another solution for the differential equation set is $\left(\left(K_{\mathrm{G}}-J_{\mathrm{G}}+R_{\mathrm{NG}}\right) /\left(K_{\mathrm{G}}+M_{\mathrm{NG}}+R_{\mathrm{NG}}\right),\left[M_{\mathrm{C}}+(1-\alpha)\right.\right.$. $\left.\left.T_{\mathrm{C}}+J_{\mathrm{NC}}\right] /\left(M_{\mathrm{C}}+R_{\mathrm{C}}+K_{\mathrm{NC}}\right)\right)$.

3.2. Stability Analysis of Equilibrium Points. To judge the stability of the equilibrium points, the Jacobian matrix of the dynamic game system needs to be calculated. The proportion of the lane changing strategy for the bus is written as $x$ to represent the general situation. When $\alpha=1, x=x_{1}$; when $\alpha=0, x=x_{2}$; when $\alpha$ is between 0 and $1, x=x_{3}$. The Jacobian matrix can be expressed as

$$
\begin{aligned}
\text { Jacobi }= & {\left[\begin{array}{ll}
\frac{F_{\mathrm{BPR}}(x, y)}{\partial x} & \frac{F_{\mathrm{BPR}}(x, y)}{\partial y} \\
\frac{F_{\mathrm{HDS}}(x, y)}{\partial x} \frac{F_{\mathrm{HDS}}(x, y)}{\partial y}
\end{array}\right] } \\
= & {\left[\begin{array}{cc}
(1-2 x)\left[y \cdot\left(M_{\mathrm{C}}+R_{\mathrm{C}}+K_{\mathrm{NC}}\right)-M_{\mathrm{C}}-(1-\alpha) \cdot T_{\mathrm{C}}-J_{\mathrm{NC}}\right] & x(1-x)\left(M_{\mathrm{C}}+R_{\mathrm{C}}+K_{\mathrm{NC}}\right) \\
y(1-y)\left(K_{\mathrm{G}}+M_{\mathrm{NG}}+R_{\mathrm{NG}}\right) & (1-2 y)\left[x \cdot\left(K_{\mathrm{G}}+M_{\mathrm{NG}}+R_{\mathrm{NG}}\right)+J_{\mathrm{G}}-K_{\mathrm{G}}-R_{\mathrm{NG}}\right]
\end{array}\right] . }
\end{aligned}
$$

Then, the determinant and trace of the Jacobian matrix, which are denoted by Det(Jacobi) and $\operatorname{Tr}$ (Jacobi), can be calculated as

$$
\begin{aligned}
\operatorname{Det}(\text { Jacobi })= & (1-2 x)(1-2 y)\left[y \cdot\left(M_{\mathrm{C}}+R_{\mathrm{C}}+K_{\mathrm{NC}}\right)-M_{\mathrm{C}}-(1-\alpha) \cdot T_{\mathrm{C}}-J_{\mathrm{NC}}\right] \times\left[x \cdot\left(K_{\mathrm{G}}+M_{\mathrm{NG}}+R_{\mathrm{NG}}\right)\right. \\
& \left.+J_{\mathrm{G}}-K_{\mathrm{G}}-R_{\mathrm{NG}}\right]-x y(1-x)(1-y)\left(M_{\mathrm{C}}+R_{\mathrm{C}}+K_{\mathrm{NC}}\right)\left(K_{\mathrm{G}}+M_{\mathrm{NG}}+R_{\mathrm{NG}}\right), \\
\operatorname{Tr}(\text { Jacobi })= & (1-2 x)\left[y \cdot\left(M_{\mathrm{C}}+R_{\mathrm{C}}+K_{\mathrm{NC}}\right)-M_{\mathrm{C}}-(1-\alpha) \cdot T_{\mathrm{C}}-J_{\mathrm{NC}}\right]+(1-2 y)\left[x \cdot\left(K_{\mathrm{G}}+M_{\mathrm{NG}}+R_{\mathrm{NG}}\right)\right. \\
& \left.+J_{\mathrm{G}}-K_{\mathrm{G}}-R_{\mathrm{NG}}\right] .
\end{aligned}
$$

Based on equations (13) and (14), the determinant and trace of every equilibrium point in the three models which corresponds to the Jacobian matrix can be solved, as listed in Table 4.

Each equilibrium point may be an evolutionary stable strategy (ESS), a saddle point, an unstable point, or a center point. The stability of each equilibrium point is related to its positive or negative value which corresponds to the determinant and trace of the Jacobian matrix. Based on the determinant and trace of the equilibrium points listed in Table 4, it can be found that the stability of the equilibrium points is determined by the relative relationship between $J_{\mathrm{NC}}-K_{\mathrm{NC}}$ and $R_{\mathrm{C}}-(1-\alpha) \cdot T_{\mathrm{C}}$ and that between $J_{\mathrm{G}}-K_{\mathrm{G}}$ and $R_{\mathrm{NG}}$. The stability of the equilibrium points in different conditions is discussed in Table 5.

From Table 5, it can be revealed that (1) when $J_{\mathrm{NC}}-K_{\mathrm{NC}}<R_{\mathrm{C}}-(1-\alpha) \cdot T_{\mathrm{C}}$, the equilibrium point $(1,1)$ is ESS; (2) when $J_{\mathrm{G}}-K_{\mathrm{G}}<R_{\mathrm{NG}}$, the equilibrium point $(0,0)$ is 


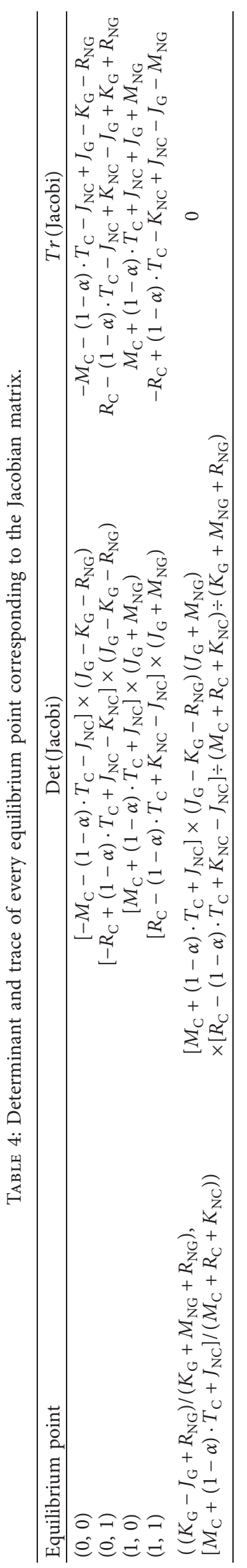




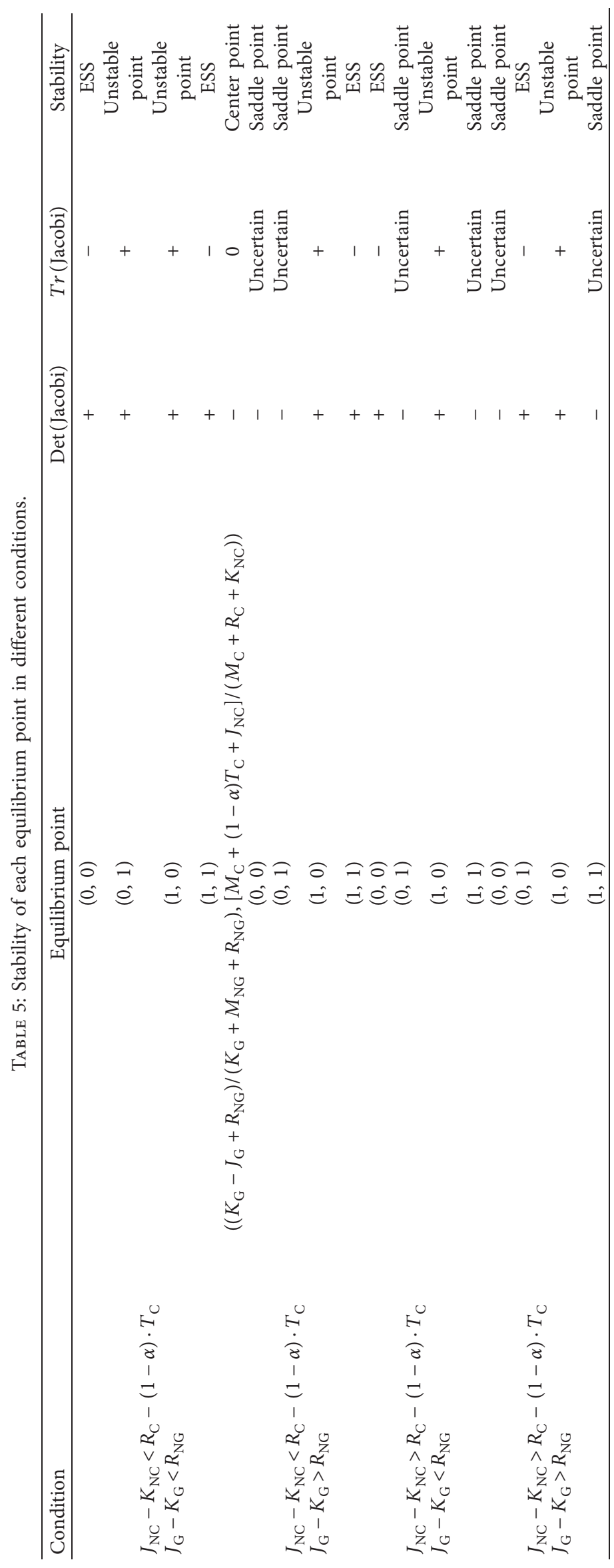


ESS; (3) when $J_{\mathrm{NC}}-K_{\mathrm{NC}}>R_{\mathrm{C}}-(1-\alpha) \cdot T_{\mathrm{C}} \quad$ and $J_{\mathrm{G}}-K_{\mathrm{G}}>R_{\mathrm{NG}}$, the equilibrium point $(0,1)$ is ESS; (4) in any condition, the determinant and trace of the Jacobian matrix for the equilibrium point $(1,0)$ are always greater than zero, that is to say, the equilibrium point $(1,0)$ is never possible to be ESS; and (5) when $J_{\mathrm{NC}}-K_{\mathrm{NC}}<R_{\mathrm{C}}-(1-\alpha) \cdot T_{\mathrm{C}}$ and $J_{\mathrm{G}}-K_{\mathrm{G}}<R_{\mathrm{NG}}$, the center point of the dynamic game system is the Nash equilibrium point of the mixed strategies.

In addition, when $J_{\mathrm{NC}}-K_{\mathrm{NC}}$ equals to $R_{\mathrm{C}}-(1-\alpha) \cdot T_{\mathrm{C}}$ or $J_{\mathrm{G}}-K_{\mathrm{G}}$ equals to $R_{\mathrm{NG}}$, each of the equilibrium points $(0$, $0),(0,1)$, and $(1,1)$ may be a saddle point or an unstable point. The evolutionary stability under these conditions is not discussed in detail in Table 5 due to the lack of significance from the perspective of providing feasible strategies for drivers and vehicles.

\section{Numerical Experiments}

4.1. Design of Numerical Experiments. The changes of all the variables except $M_{\mathrm{C}}$ and $M_{\mathrm{NG}}$ will cause the changes of conditions in the stability analysis, as listed in Table 5, which will lead to different evolutionary results. However, the changes of $M_{\mathrm{C}}$ and $M_{\mathrm{NG}}$ cannot be revealed directly in the stability analysis. It makes sense to discuss the sensitivities of the proportions of the strategies (i.e., the proportion of lanechanging strategy for the bus and that of giving way strategy for HDS) to the changes of $M_{\mathrm{C}}$ and $M_{\mathrm{NG}}$. Moreover, different initial proportions of the strategies for different populations will affect the results of dynamic evolution, and it will be analyzed in the following. Meanwhile, when the penetration rate of the connected autonomous bus varies, traffic flow will display different characteristics, which will influence the direction of evolution. Therefore, it has great significance to research the impacts of different penetration rates of the connected autonomous bus on the evolutionary results.

Besides the influences of the change of a single variable on the proportions of the strategies, the effects of the changes of a set of variables on the proportions of the strategies should be also noted. Through the above analysis about the stability of the equilibrium points, it can be found that each judgement condition is the difference between the payoff of a safer strategy and that of a more efficient strategy. Such a difference determines the condition of the stability of each equilibrium point and will have an effect on the dynamic game system. For the bus, the difference is the payoff of not changing lanes minus that of changing lanes when HDS gives way, that is, $J_{\mathrm{NC}}-K_{\mathrm{NC}}-R_{\mathrm{C}}$ in Model $1, J_{\mathrm{NC}}-K_{\mathrm{NC}}-$ $\left(R_{\mathrm{C}}-T_{\mathrm{C}}\right)$ in Model 2 , and $J_{\mathrm{NC}}-K_{\mathrm{NC}}-\left[R_{\mathrm{C}}-(1-\alpha) \cdot T_{\mathrm{C}}\right]$ in Model 3, respectively, and such a difference is defined as $\Delta B$. For HDS, the difference is the payoff of giving way minus that of not giving way when the bus does not change lanes, that is, $J_{\mathrm{G}}-K_{\mathrm{G}}-R_{\mathrm{NG}}$, and such a difference is denoted by $\Delta S$.

To carry out the numerical experiments, the initial value of every variable should be given. Compared with HDS, whether the bus is $\mathrm{CAB}$ or $\mathrm{HDB}$, there are more passengers on the bus, and the bus will gain more time benefit or lose more time loss. Therefore, $R_{\mathrm{C}}>R_{\mathrm{NG}}$, and $K_{\mathrm{NC}}>K_{\mathrm{G}}$.
Meanwhile, when the driver or vehicle takes a conservative action (i.e., not changing lanes for the bus and giving way for HDS), more time is needed for rereacting and restarting. The time loss for the vehicle is more than its time benefit in this case, so $K_{\mathrm{NC}}>R_{\mathrm{C}}$ and $K_{\mathrm{G}}>R_{\mathrm{NG}}$. Specifically, $T_{\mathrm{C}}, J_{\mathrm{NC}}$, and $R_{\mathrm{NG}}$ are all set to $3, M_{\mathrm{C}}$ is set to $4, R_{\mathrm{C}}, K_{\mathrm{G}}, J_{\mathrm{G}}$, and $M_{\mathrm{NG}}$ are all set to 5 , and $K_{\mathrm{NC}}$ is set to 7 . As for the initial penetration rate, $\alpha$ is set to 0.5 in order to adapt the future development of connected autonomous vehicles in China [34]. According to the results listed in Table 5, the current variable values may lead to two stable states, i.e., $(0,0)$ and $(1,1)$. The initial values of $x_{1}, x_{2}$, and $x_{3}$ are all set to 0.7 , and the initial value of $y$ is set to 0.3 , respectively. In this case, it seems that the risk of collision will increase for the bus and HDS. With the increased collision risk, the evolution direction of the dynamic game system needs to be discussed to ensure safety in the process of lane changing. Then, when the initial values of the proportions of the strategies are friendly for both the populations in the game to cooperate, safety can be guaranteed even more. It should be noted that when the sensitivities of the evolutionary results to the specific variable are analyzed, the value of the specific variable will be determined.

4.2. Impacts of Safety Loss on Evolution. Based on the initial values predetermined above, the safety loss of changing lanes for the bus, which is denoted by $M_{C}$, is set to 0,4 , and 8 , respectively. The evolutionary paths of the proportion of the lane-changing strategy under different safety losses in the three models are shown in Figure 2.

As illustrated in Figure 2(a), the convergence rate of the proportion of the lane-changing strategy for $\mathrm{CAB}$ slows down as $M_{\mathrm{C}}$ increases, but the stable strategy for $\mathrm{CAB}$ is always changing lanes. It is indicated that the increasing safety loss has no influence on the final decision-making for $\mathrm{CAB}$, and person delay caused by mass passengers carried by $\mathrm{CAB}$ will be efficiently reduced. Compared with $\mathrm{CAB}, \mathrm{HDB}$ shows conservative tendencies in the strategy evolutions, as displayed in Figure 2(b). Specifically, the convergence time of the proportion of lane-changing strategy for HDB is obviously longer than that for CAB. When the safety loss is great to changing lanes, $\mathrm{HDB}$ is very likely to choose the strategy of not changing lanes. Besides safety loss, extra comfort loss may lead HDB to choose the strategy of not changing lanes to avoid risks in lane changing. As shown in Figure 2(c), when $\mathrm{CAB}$ and $\mathrm{HDB}$ are mixed, the evolutionary paths reveal the same trends with those in Model 1, but the convergence in Model 3 is slightly slower than that in Model 1. Through replacing a part of $\mathrm{CAB}$ with $\mathrm{HDB}$, the aggressive tendencies of $\mathrm{CAB}$ are weakened, as shown in Figures 2(a) and 2(c).

The safety loss of not giving way for HDS, which is denoted by $M_{\mathrm{NG}}$, is set to 1,5 , and 9 , respectively. The evolutionary paths of the proportion of giving way strategy under different safety losses in the three models are shown in Figure 3. As shown in Figure 3(a), even if the safety loss is relatively small for HDS, it still chooses to give way because of the smaller time loss, compared with CAB. In this case, the 

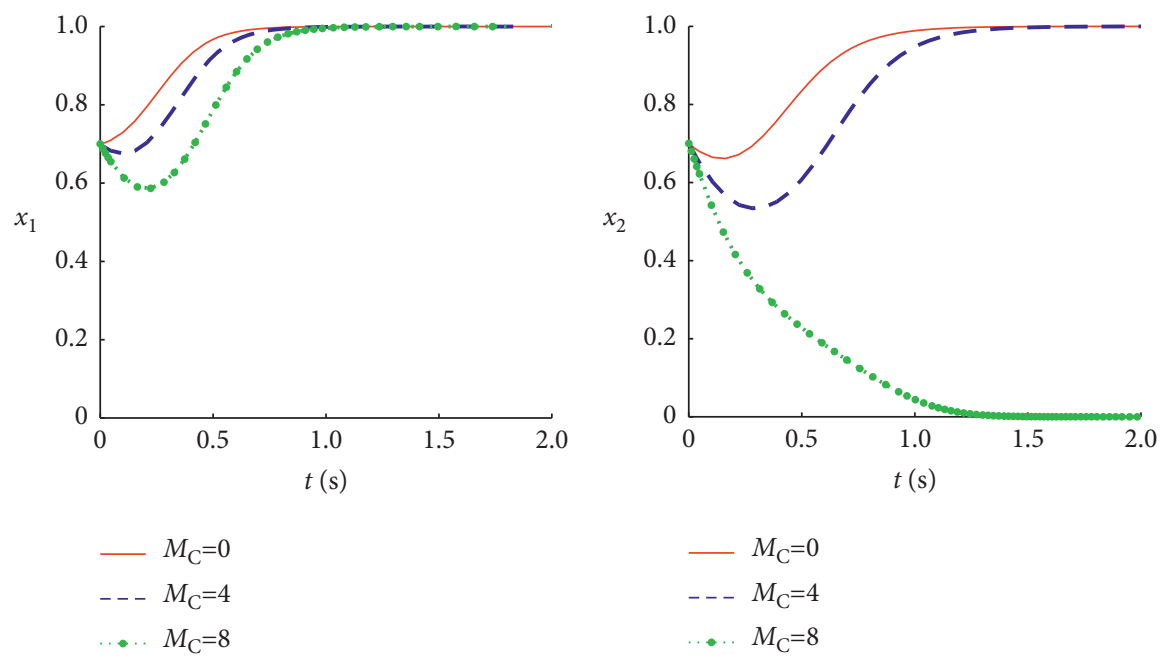

(a)

(b)

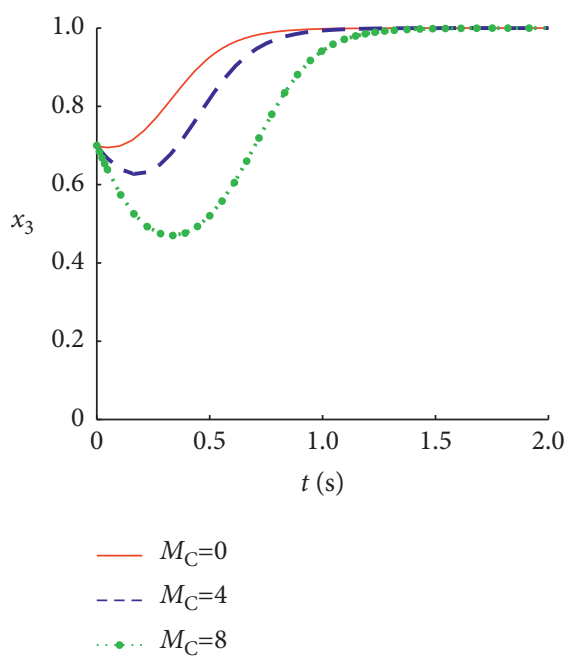

(c)

FIGURE 2: Evolutionary paths of the proportion of lane changing strategy under different safety losses. (a) Model 1; (b) Model 2; (c) Model 3.

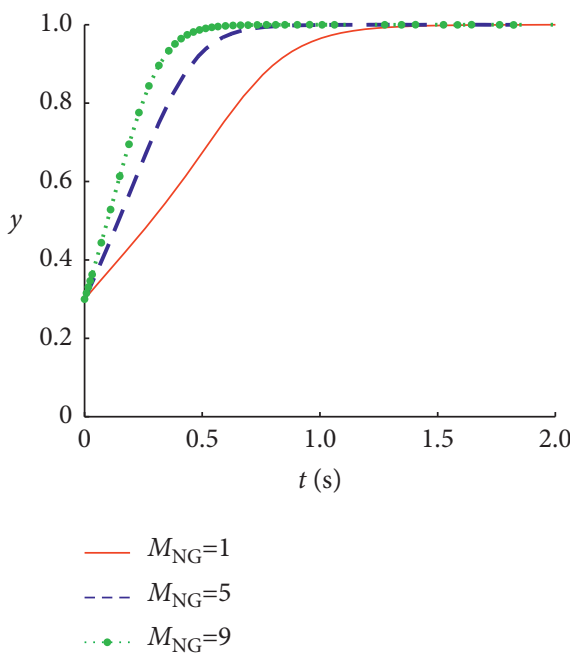

(a)

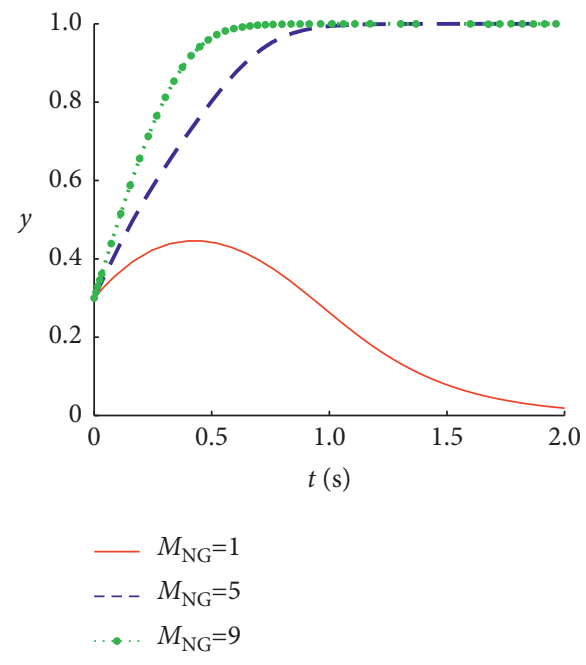

(b)

FIGURE 3: Continued. 


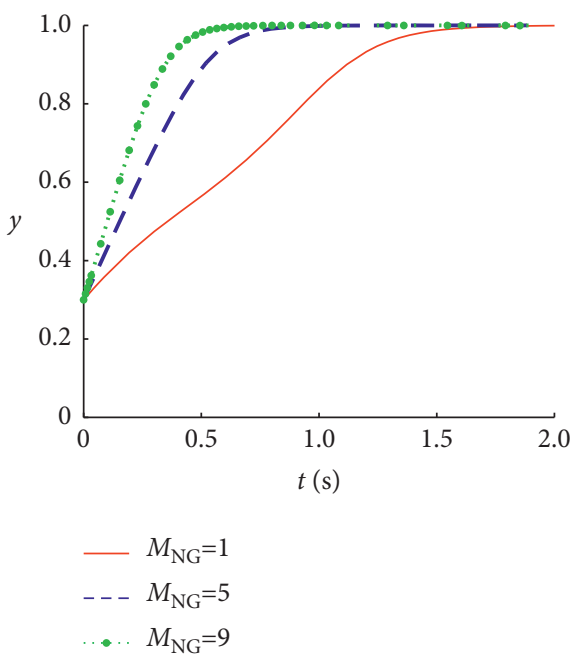

(c)

Figure 3: Evolutionary paths of the proportion of giving way strategy under different safety losses. (a) Model 1; (b) Model 2; (c) Model 3.

stable state of changing lanes and giving way is easily formed. The evolutionary path of the proportion of giving way strategy in Model 2 shows different characteristics, as displayed in Figure 3(b). When the safety loss is very small, the proportion of giving way strategy shows a trend of rising first and then falling, and finally the aggressive strategy of not giving way is more favorable for HDS. With the safety loss increasing, the proportion of giving way strategy converges to 1 . The larger the safety loss is, the faster the convergence is. That is to say, increasing the safety loss promotes the cooperation of social vehicles. As presented in Figure 3(c), the evolutionary paths in Model 3 show the same trends with those in Model 1, that is, the increasing safety loss of not giving way promotes the selection of the strategy of giving way.

Based on the parameter values and the above analyses, there are only two stable states, i.e., $(0,0)$ and $(1,1)$. The stable state $(1,1)$ for the bus and HDS exists in most instances with different safety losses, and in the game between HDB and HDS, the stable state $(0,0)$ may exist. More time will lose in the stable state $(0,0)$ from the overall efficiency viewpoint because of the mass passengers carried by the bus, even if the efficiency of HDS is guaranteed. Therefore, it is necessary to cultivate the social vehicle drivers' habit of courtesy and their overall concept of traffic delays so as to obtain the stable state $(1,1)$. Furthermore, the social vehicle drivers' risk awareness should be improved because they will suffer economic loss besides safety loss if the social vehicles have collisions with the buses.

\subsection{Impacts of Initial Proportions of Strategies on Evolution.} To discuss the sensitivity of ESS to the initial proportion of the lane-changing strategy, the initial value of the proportion of lane-changing strategy, which is denoted by $x_{0}$, is set to $0.1,0.3,0.5,0.7$, and 0.9 , respectively. The initial value of the proportion of giving way strategy is set to 0.3 based on the above analyses. The evolutionary paths of the proportion of the lane-changing strategy under different initial proportions are illustrated in Figure 4.

As shown in Figure 4, except for the case of $x_{0}=0.5$, the evolutions display the same trends in different scenarios, and the stable strategies are related to the initial proportions. Specifically, when the initial proportion of the lane-changing strategy equals to 0.1 or 0.3 , the stable strategy for the bus is not to change lanes; when the initial proportion equals to 0.7 or 0.9 , the dynamic game system stables at the strategy of changing lanes. As the strategies of changing lanes and not changing lanes are evenly mixed at the beginning, different characteristics are revealed in the three models. In Models 1 and 3 , the stable strategy is to change lanes, but more time will be needed in Model 3 to obtain the stable state. The evolution result in Model 2 is the opposite; that is, HDB is inclined to the strategy of not changing lanes because of extra comfort loss, which further indicates the conservative tendencies of HDB.

The initial value of the proportion of giving way strategy, which is denoted by $y_{0}$, is set to $0.1,0.3,0.5,0.7$, and 0.9 , respectively. The initial value of the proportion of lanechanging strategy is set to 0.7 . The evolutionary paths of the proportion of giving way strategy under different initial proportions are illustrated in Figure 5.

Generally, the evolutionary paths in Models 1, 2, and 3 are similar, and the cooperative tendency of HDS is indicated in most instances. The stable strategies for HDS are to give way under different initial proportions excluding the case of $y_{0}=0.1$. The higher the initial proportion is, the faster the convergence is. When $y_{0}=0.1$, the proportion of giving way strategy stables at 0 . Compared with the convergence of the proportions of the strategies in Models 2 and 3 , longer time, i.e., almost 4 seconds, is needed in Model 1 to converge to 0 . The strategies of giving way for HDS should be encouraged in a connected and autonomous driving environment to improve traffic efficiency.

It seems that it is conductive to the formation of the stable state $(1,1)$ via maximizing the proportion of the lane- 


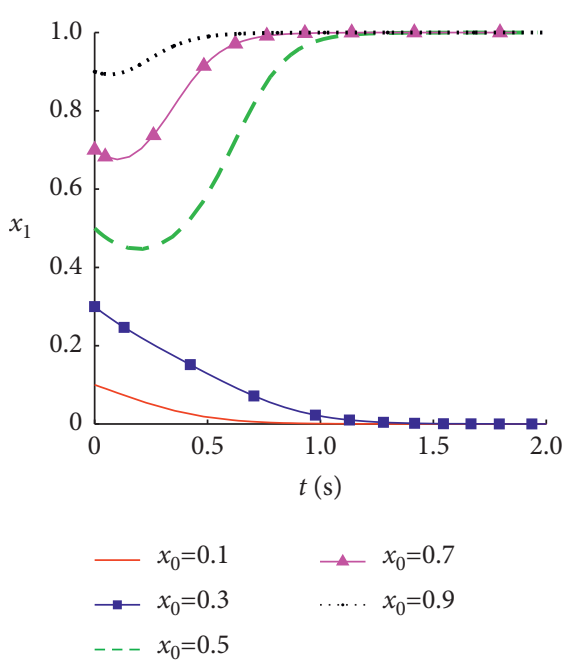

(a)

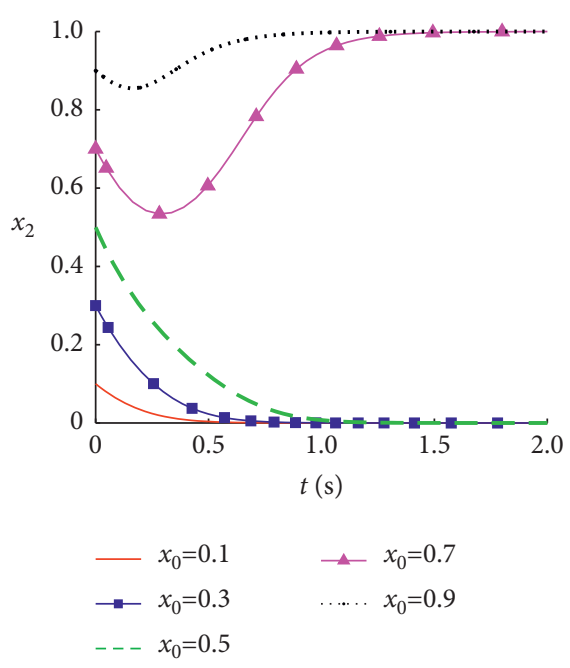

(b)

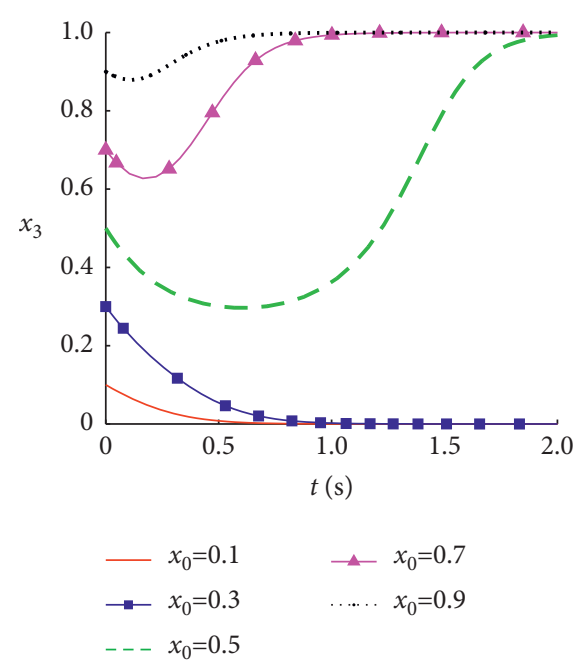

(c)

FIGURE 4: Evolutionary paths of the proportion of lane changing strategy under different initial proportions. (a) Model 1; (b) Model 2; (c) Model 3.

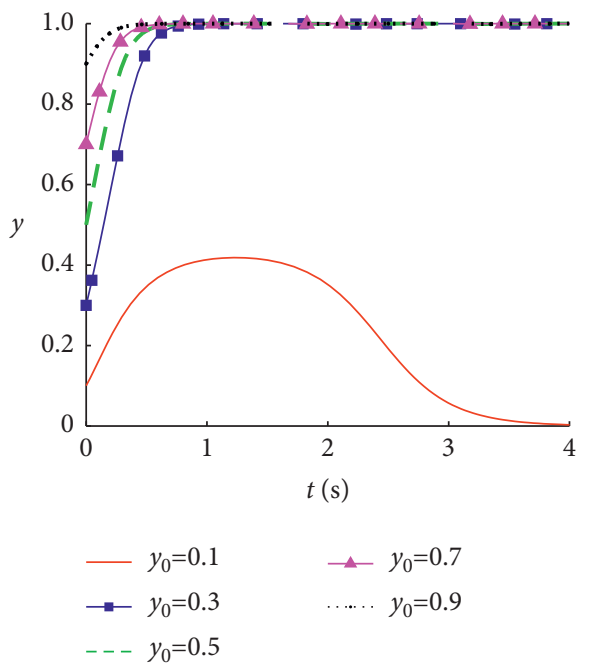

(a)
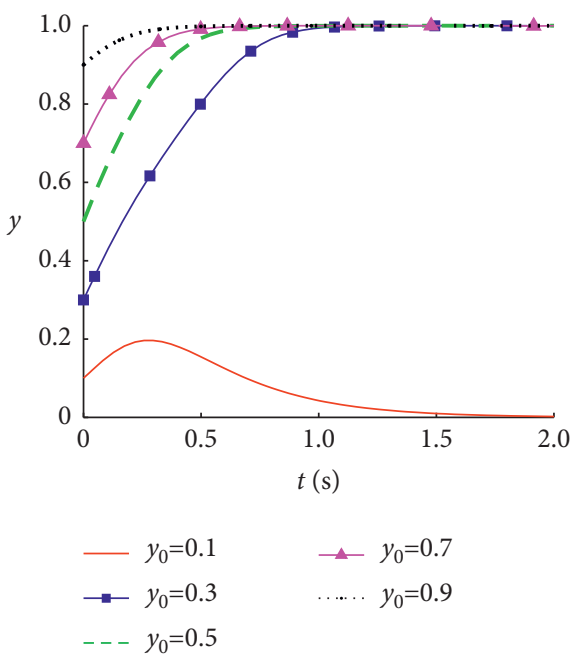

(b)

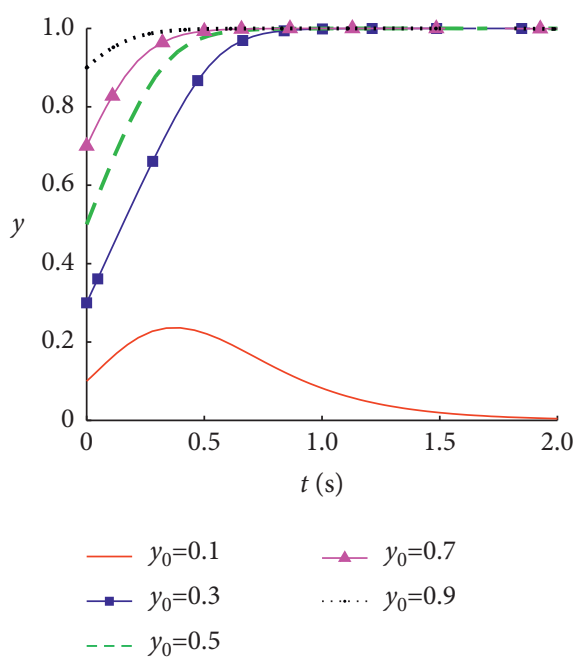

(c)

FIGURE 5: Evolutionary paths of the proportion of giving way strategy under different initial proportions. (a) Model 1; (b) Model 2; (c) Model 3. 
changing strategy. However, such an approach is harmful to traffic safety, especially for HDB because of the extra comfort loss caused by the noncollision injuries. In this case, turning on the signal lights in time should be encouraged in order to indicate the tendency of changing lanes and seek the cooperation of HDS, and the clear signal to giving way needs to be shown by HDS. Meanwhile, traffic managers need to formulate relevant management measures, such as setting up suitable parking places for giving way around the bus bay stop, and giving HDS a corresponding penalty if HDS does not give way, when the bus shows a clear tendency of changing lanes.

\subsection{Impacts of the Penetration Rate of Connected Autonomous} Bus on Evolution. The changes of the penetration rate of the connected autonomous bus and safety loss are both considered here to explore the sensitivities of the proportions of the strategies to the penetration rate of the connected autonomous bus comprehensively in different situations. Under different penetration rates, there are always two stable states, i.e., $(0,0)$ and $(1,1)$. The value of safety loss is the same as that in Section 4.2. The evolutionary convergence of the proportion of lane-changing strategy under different penetration rates of the connected autonomous bus is shown in Figure 6.

From Figure 6, it can be found that (1) as $\alpha$ increases, no matter what the value of safety loss is, the convergence of the proportion of lane changing strategy becomes faster; (2) with the growth of safety loss, the conservative tendencies of HDB are gradually shown, and the specific performance is that more time is needed for the convergence of the stable strategy of changing lanes, and the evolutionary trends of falling first and then rising become more obvious; and (3) when $M_{\mathrm{C}}=8$ and $\alpha$ is small, the conservative tendencies of HDB are fully reflected, that is, the evolution stables at the strategy of not changing lanes, and the smaller the penetration rate of the connected autonomous bus is, the faster the convergence is.

The evolutionary convergence of the proportion of giving way strategy under different penetration rates of the connected autonomous bus is shown in Figure 7. It can be revealed that (1) as $\alpha$ increases, no matter what the value of safety loss is, the rate at which the proportion of giving way strategy converges to 1 increases; (2) as the safety loss increases, the evolutionary paths keep rising, and the convergence of the proportion of giving way strategy becomes faster, that is to say, the strategy of giving way is more beneficial to obtain the Nash equilibrium; and (4) when $M_{\mathrm{NG}}=1$ and $\alpha$ is small, the aggressive strategy of not giving way for HDS is the best response to the strategy for the bus, and the smaller the penetration rate of the connected autonomous bus is, the faster the convergence is.

Hence, through the improvement of vehicle intelligence and networking, overall traffic efficiency will be rising. Meanwhile, the drop of the safety loss of changing lanes and the increase of the safety loss of not giving way will be helpful to enhance overall traffic efficiency. It is essential for traffic managers to make the HDS driver fully aware of the safety loss caused by not giving way through driver safety training, traffic guidance, traffic penalty, etc. In addition, safety risks in the lane changing for the bus, including the collision and noncollision risks, should be minimized as much as possible in the design and operation for the bus.

4.5. Impacts of $\Delta B$ and $\Delta$ S on Evolution. As defined above, $\Delta B$ and $\Delta S$ are the differences between a safer strategy and a more efficient strategy for the bus and HDS, respectively. The sensitivities of the proportions of the strategies to $\Delta B$ and $\Delta S$ under different penetration rates of the connected autonomous bus are discussed here. The penetration rates of the connected autonomous bus are set to $0,0.5$, and 1 , respectively, to make the changing trends as clear as possible. In order to discover the changing process of the two stable states in the process of evolution, i.e., change lanes and give way and do not change lanes and do not give way, $\Delta B$ is set to -5 to 5 . The evolutionary convergence of the proportion of the lane-changing strategy under different $\Delta B$ values is displayed in Figure 8.

From Figure 8, the results reveal that (1) with the growth of $\Delta B$, the proportion of the lane-changing strategy gradually changes from converging to 1 to converging to 0 , and the safer strategy of not changing lanes is more favorable for the bus; (2) as $\alpha$ increases, the critical point of $\Delta B$ becomes larger, and it means that a part of the strategy of not changing lanes is replaced by the strategy of changing lanes; and (3) as the penetration rate of the connected autonomous bus increases, the convergence of the strategy of not changing lanes becomes slower and that of changing lanes becomes faster, which further indicates that when more CABs are used, the more efficient strategy is conductive to the stability of the dynamic game system.

Similarly, $\Delta S$ is set to -15 to -5 to fully describe the transformation of the two stable states, that is, the stable states of changing lanes and giving way, and those of not changing lanes and not giving way. The evolutionary convergence of the proportion of giving way strategy under different $\Delta S$ values is illustrated in Figure 9. It can be found that (1) as $\Delta S$ grows, the proportion of the giving way strategy changes from converging to 0 to converging to 1 , and HDS is more inclined to the giving way strategy; (2) with the increase of $\alpha$, the critical point of $\Delta S$ becomes smaller, which implies that the safer strategy is helpful for HDS to get the stable states; and (3) as $\alpha$ increases, the convergence of the giving way strategy grows and that of not giving way strategy decreases, and the tendency of the safer strategy for HDS is further explained.

When the difference between a safer strategy and a more efficient strategy becomes larger, the two populations are both more inclined to choose a safer strategy in the evolutionary game, that is, not changing lanes for the bus and giving way for HDS. With the increase of $\alpha$, the dynamic game system evolves in the direction of optimal overall traffic efficiency under the premise of ensuring safety. Therefore, compared with the efficient strategy, the strategy that is related to traffic safety is more important in the lane changing game for exiting for the connected autonomous bus. In the development and practice of connected 


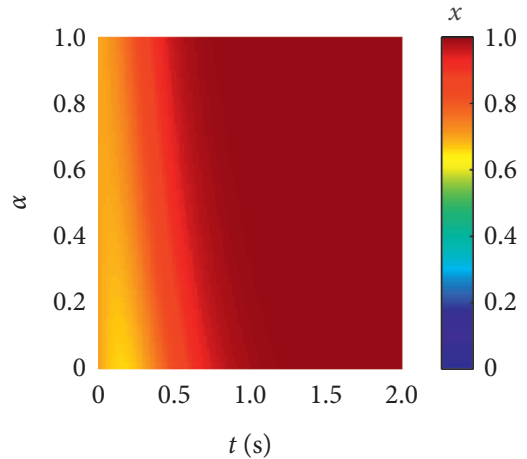

(a)

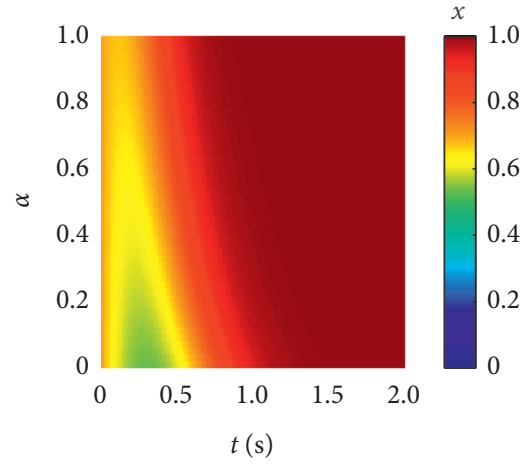

(b)

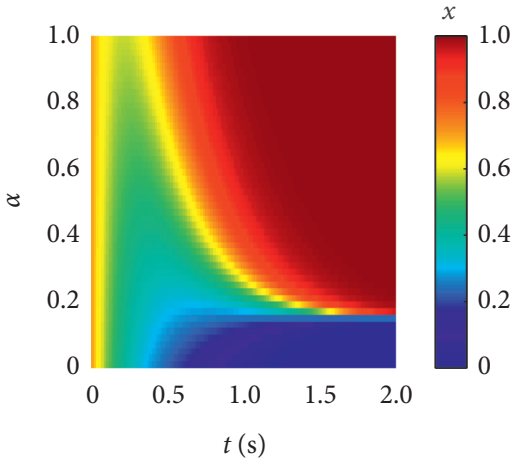

(c)

Figure 6: Evolutionary convergence of the proportion of lane changing strategy under different penetration rates of the connected autonomous bus. (a) $M_{\mathrm{C}}=0$; (b) $M_{\mathrm{C}}=4$; (c) $M_{\mathrm{C}}=8$.

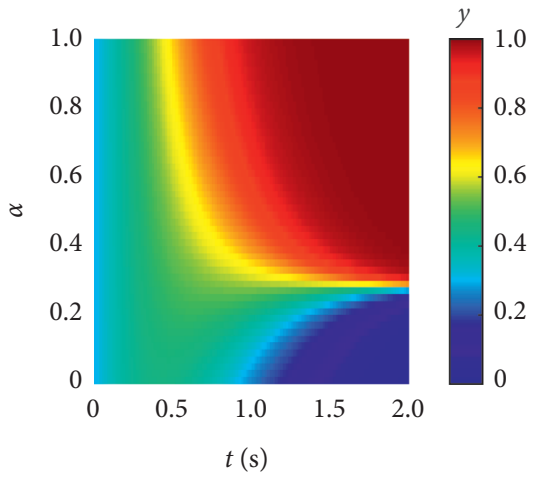

(a)

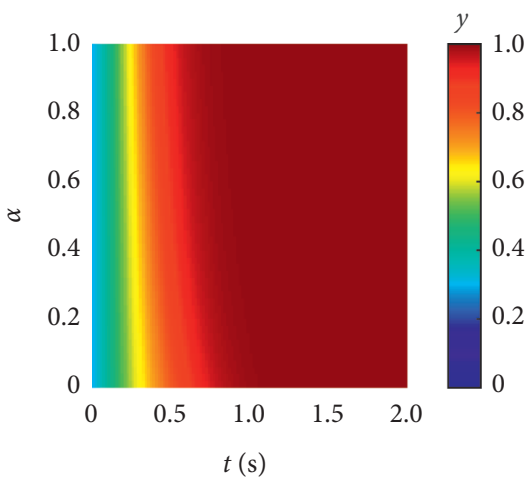

(b)

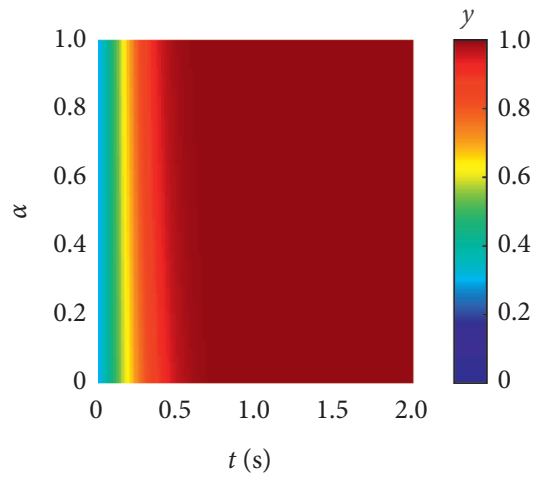

(c)

FiguRE 7: Evolutionary convergence of the proportion of giving way strategy under different penetration rates of the connected autonomous bus. (a) $M_{\mathrm{NG}}=1$; (b) $M_{\mathrm{NG}}=5$; (c) $M_{\mathrm{NG}}=9$.

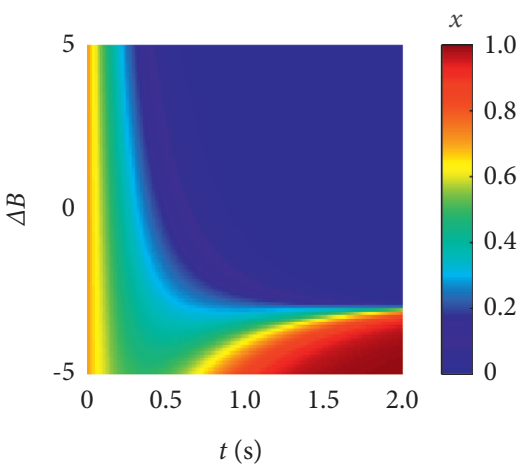

(a)

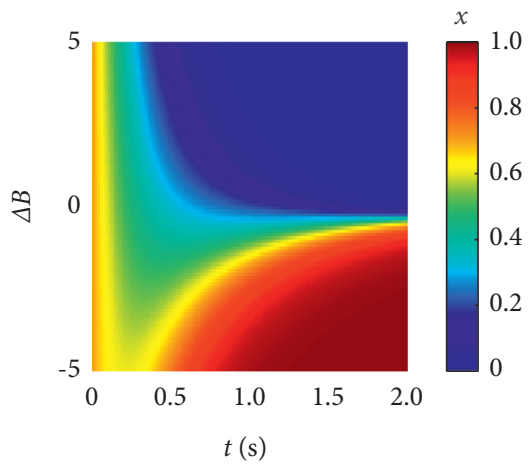

(b)

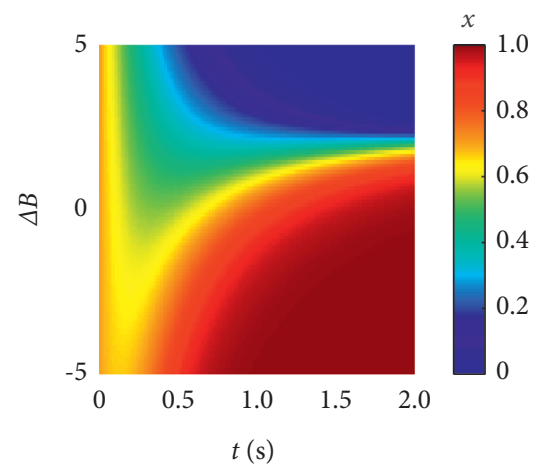

(c)

FIGURE 8: Evolutionary convergence of the proportion of lane changing strategy under different $\Delta B$ values. (a) $\alpha=0$; (b) $\alpha=0.5$; (c) $\alpha=1$.

autonomous vehicles, traffic safety should be the first concern. Through the intelligent control of driving assistance systems in vehicles, especially adjusting the parameters related to passenger delays, the negative impacts of lane changing on traffic efficiency can be eliminated after driving safety is guaranteed. 


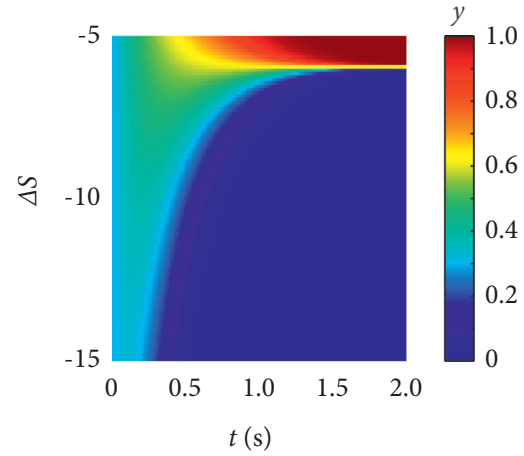

(a)

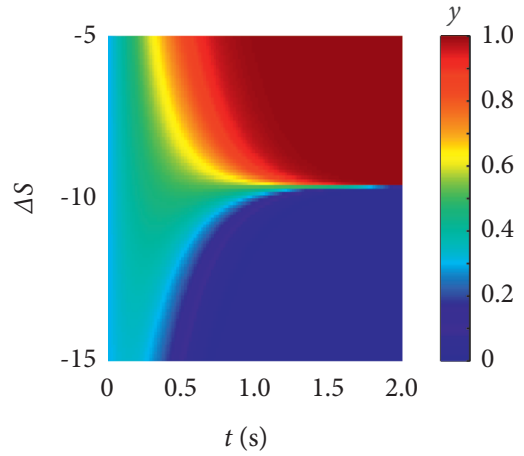

(b)

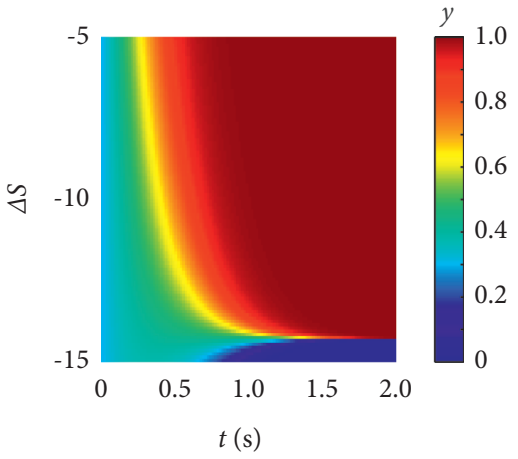

(c)

FIGURE 9: Evolutionary convergence of the proportion of giving way strategy under different $\Delta S$ values. (a) $\alpha=0$; (b) $\alpha=0.5$; (c) $\alpha=1$.

\section{Conclusions}

In this study, the mandatory lane-changing issue for bus exiting is modeled by applying the evolutionary game theory. Specifically, the evolutionary game models for $\mathrm{CAB}$ and HDS, HDB and HDS, and BPR and HDS are formulated, respectively, in order to analyze the evolutionary dynamics under different scenarios. The payoff matrices of the bus and social vehicle are constructed mainly from the perspectives of the safety benefit, safety loss, time benefit, and time loss. Moreover, the passengers on HDB will suffer the comfort loss caused by the lack of smoothness of start acceleration, so the comfort loss is added in the payoff matrix of the game between HDB and HDS. Based on the payoff matrices, the replicator dynamics equations for the three models are deduced. Then, the equilibrium points of the dynamic game system are calculated according to the differential equations that are constructed by the replicator dynamics equations for different populations. Through analyzing the determinant and trace of the Jacobian matrix for different equilibrium points in different conditions, the stability of the equilibrium points is described. There are several cases of stability in the dynamic game system, including the ESS, saddle point, unstable point, and center point.

According to the game models under different scenarios, numerical experiments are carried out. The experimental design is focused on the sensitivities of the proportions of the strategies to the safety loss, initial proportion, and the penetration rate of the connected autonomous bus. Meanwhile, the sensitivities of the proportions of the strategies to a set of variables, i.e., the difference between the payoff of a safer strategy and that of a more efficient strategy, are taken into account. The simulation findings imply that, in most cases, the dynamic game system converges to $(1,1)$ with the initial values of the variables considering the characteristic of traffic flow. But there are several cases where the system converges to $(0,0)$, for example, large safety loss of changing lanes for the bus and small initial proportions of the strategies for the bus and social vehicle. Furthermore, compared with $\mathrm{CAB}$, the evolutionary stable state of $\mathrm{HDB}$ is more sensitive to the change in safety loss and shows a conservative tendency. In the game between BPR and social vehicle, the dynamic game system is likely to stabilize in the strategy profile that takes into account both overall traffic efficiency and safety. The larger the penetration rate is, the faster the convergence is. Some suggestions that are related to traffic management and driving behaviors are provided to help to mitigate the conflicts between the bus and social vehicle in the lane-changing process.

The models proposed in this paper will be effective to improve overall traffic efficiency through changing the parameters in the payoff matrices so as to adjust the evolution direction of the dynamic game system. In the era of connected autonomous vehicles, driving safety is the most concerned issue for traffic researchers, designers, and users. The motivation of cooperative strategies is of great significance to help to avoid conflicts among vehicles. For future research, the connected autonomous social vehicle and its different penetration rates are both worth considering in the evolutionary game models of lane changing for bus exiting.

\section{Data Availability}

The values of the parameters are given in the submitted article. The data from figures are simulated using MATLAB software to solve the evolutionary game models.

\section{Conflicts of Interest}

The authors declare that they have no conflicts of interest.

\section{Acknowledgments}

The research reported in this article has been funded by the National Natural Science Foundation of China (Grant no. 52172314) and the Fundamental Research Funds for the Central Universities of Ministry of Education of China (Grant no. DUT20JC40).

\section{References}

[1] Y. Bie, J. Ji, X. Wang, and X. Qu, "Optimization of electric bus scheduling considering stochastic volatilities in trip travel time and energy consumption," Computer-Aided Civil and Infrastructure Engineering, vol. 36, 2021 (In press). 
[2] Y. Bie, M. Hao, and M. Guo, "Optimal electric bus scheduling based on the combination of all-stop and short-turning strategies," Sustainability, vol. 13, no. 4, p. 1827, 2021.

[3] H. Kita, "A merging-giveway interaction model of cars in a merging section: a game theoretic analysis," Transportation Research Part A: Policy and Practice, vol. 33, no. 3-4, pp. 305-312, 1999.

[4] D. Arbis and V. V. Dixit, "Game theoretic model for lane changing: incorporating conflict risks," Accident Analysis \& Prevention, vol. 125, pp. 158-164, 2019.

[5] J. Peng, Y. Guo, and Y. Shao, "Lane change decision analysis based on drivers' perception-judgment and game theory," Applied Mechanics and Materials, vol. 361-363, pp. 18751879, 2013.

[6] K. Kang and H. A. Rakha, "Game theoretical approach to model decision making for merging maneuvers at freeway onramps," Transportation Research Record: Journal of the Transportation Research Board, vol. 2623, no. 1, pp. 19-28, 2017.

[7] M. ZimmermannD. Schopf et al., "Carrot and stick: a gametheoretic approach to motivate cooperative driving through social interaction," Transportation Research Part C: Emerging Technologies, vol. 88, pp. 159-175, 2018.

[8] H. Shao, J. Wang, Y. Wang, and S. Chen, "Electric bicycle lane-changing behavior under strategy games," Sustainability, vol. 10, no. 9, 2018.

[9] X. Ba and J. Liu, "Research on decision making behavior of bus entering lane-changing based on game theory," Journal of Chongqing University of Technology (Natural Science), vol. 33, no. 2, pp. 111-116, 2019.

[10] K. Kang and H. A. Rakha, "Modeling driver merging behavior: a repeated game theoretical approach," Transportation Research Record: Journal of the Transportation Research Board, vol. 2672, no. 20, pp. 144-153, 2018.

[11] J. C. Harsanyi, "Games with incomplete information player by "Bayesian" players, I-III: Part I. the basic model," Management Science, vol. 50, no. 12, pp. 1804-1817, 2004.

[12] A. Talebpour, H. S. Mahmassani, and S. H. Hamdar, "Modeling lane-changing behavior in a connected environment: a game theory approach," Transportation Research Part C: Emerging Technologies, vol. 59, pp. 216-232, 2015.

[13] Y. Ali, Z. Zheng, M. M. Haque, and M. Wang, "A game theory-based approach for modelling mandatory lanechanging behaviour in a connected environment," Transportation Research Part C: Emerging Technologies, vol. 106, pp. 220-242, 2019.

[14] J. Yoo and R. Langari, "A predictive perception model and control strategy for collision-free autonomous driving," IEEE Transactions on Intelligent Transportation Systems, vol. 20, no. 11, pp. 4078-4091, 2019.

[15] H. Yu, H. E. Tseng, and R. Langari, "A human-like game theory-based controller for automatic lane changing," Transportation Research Part C: Emerging Technologies, vol. 88, pp. 140-158, 2018.

[16] D. Lin, L. Li, and S. E. Jabari, "Pay to change lanes: a cooperative lane-changing strategy for connected/automated driving," Transportation Research Part C: Emerging Technologies, vol. 105, pp. 550-564, 2019.

[17] K. Li, Y. Dai, S. Li, and M. Bian, "State-of-the-art and technical trends of intelligent and connected vehicles," Journal of automotive safety and energy, vol. 8, no. 1, pp. 1-14, 2017.

[18] M. Cui, H. Huang, Q. Xu, and J. Sekiguchi, "Survey of Intelligent and Connected Vehicle Technologies: Architectures,
Functions and Applications," Journal of Tsinghua University (Science and Technology), 2021.

[19] F. Yan, M. Liu, C. Ding, Y. Wang, and L. Yan, "Driving style recognition based on electroencephalography data from a simulated driving experiment," Frontiers in Psychology, vol. 10, 2019.

[20] D. Huo, J. Ma, and R. Chang, "Lane-changing-decision characteristics and the allocation of visual attention of drivers with an angry driving style," Transportation Research Part F: Traffic Psychology and Behaviour, vol. 71, pp. 62-75, 2020.

[21] J. W. Weibull, Evolutionary Game Theory, The MIT Press, London, UK, 1996.

[22] X. Lin and P. Yuan, "A dynamic parking charge optimal control model under perspective of commuters' evolutionary game behavior," Physica A: Statistical Mechanics and Its Applications, vol. 490, pp. 1096-1110, 2018.

[23] Q. Li, Y. Wang, K. Li, L. Chen, and Z. Wei, "Evolutionary dynamics of the last mile travel choice," Physica A: Statistical Mechanics and Its Applications, vol. 536, Article ID 122555, 2019.

[24] Z. Bai, M. Huang, S. Bian, and H. Wu, "A Study of taxi service mode choice based on evolutionary game theory," Journal of Advanced Transportation, vol. 2019, Article ID 8607942, 17 pages, 2019.

[25] L. Y. Wei, Y. F. Cui, and D. Y. Li, "Evolution mechanism of conflict between pedestrian and vehicle based on evolutionary game theory," Acta Physica Sinica, vol. 67, no. 19, Article ID 190201, 2018.

[26] F. Feng, C. Liu, and J. Zhang, “China's railway transportation safety regulation system based on evolutionary game theory and system dynamics," Risk Analysis, vol. 40, no. 10, pp. 1944-1966, 2020.

[27] K. Li, Y. Zhang, and J. Guo, "Optimization of reward and punishment mechanism for high-speed railway operation safety supervision system," Journal of transportation systems engineering and information technology, vol. 18, no. 5, pp. 18-25, 2018.

[28] A. Ji and D. Levinson, "A review of game theory models of lane changing," Transportmetrica: Transportation Science, vol. 16, no. 3, pp. 1628-1647, 2020.

[29] Y. Lwamura and J. Tanimoto, "Complex traffic flow that allows as well as hampers lane-changing intrinsically contains social-dilemma structures," Journal of Statistical Mechanics: Theory and Experiment, vol. 2018, Article ID 023408, 2018.

[30] J. Tanimoto, M. Futamata, and M. Tanaka, "Automated vehicle control systems need to solve social dilemmas to be disseminated," Chaos, Solitons \& Fractals, vol. 138, Article ID 109861, 2020.

[31] P. Bansal and K. M. Kockelman, "Forecasting Americans' long-term adoption of connected and autonomous vehicle technologies," Transportation Research Part A: Policy and Practice, vol. 95, pp. 49-63, 2017.

[32] NTCAS (National Technical Committee of Auto Standardization), "Taxonomy of driving automation for vehicles," NTCAS, Beijing, China, GB/T 40429-2021, 2019.

[33] X. Karekla and N. Tyler, "Reducing non-collision injuries aboard buses: passenger balance whilst walking on the lower deck," Safety Science, vol. 105, pp. 128-133, 2018.

[34] K. Li, "Intelligent Connected Vehicle Technology Roadmap 2.0," in Proceedings of the World Intelligent Connected Vehicles Conference, Beijjig, China, September 2020. 